\title{
Towards a quantum universe
}

\author{
Jaume Giné ${ }^{1}$
}

\begin{abstract}
In this short review we study the state of the art of the great problems in cosmology and their interrelationships. The reconciliation of these problems passes undoubtedly through the idea of a quantum universe.
\end{abstract}

Keywords Cosmology, Gravitation theory, Quantum mechanics, General relativity, large numbers, cosmological constant

\section{Introduction}

The great challenge of contemporary physics is to reconcile quantum mechanics, applied at micro cosmos, and general relativity applied, in general, at macro cosmos. General relativity and classical electrodynamics equations are invariant under a scale transformation of time intervals and distances, provided we scale too the correspondent coupling factors. In particular, the scale invariance of general relativity was applied to the strong gravity Salam \& Strathdee (1977, 1978); Caldirola et al. (1978); Sivaram \& Sinha (1979) that tries to derive the hadron properties from a scaling down of gravitational theory, treating particle as blackhole type solutions. Last years, in several works, it was suggested that also quantum mechanics must be invariant under discrete scale transformations, see Carneiro (1998). All suggest that these two irreconcilable theories, the gravity defined by the General relativity and the quantum mechanics, can be applied to any scale. Should therefore be complementary theories that explain the same physical reality.

However, the introduction of the Planck's constant $h$ in the quantum mechanics defines a very particular

Jaume Giné

Departament de Matemàtica, Universitat de Lleida,

Av. Jaume II, 69. 25001 Lleida, Spain scale, at which the quantum effects must be considered. The quantum equations, as Schrödinger and Dirac ones, are not scale invariants, due to the presence of $h$. The question that naturally arises is whether it is really a physical constant at any scale.

The invariance under discrete scale transformations appear from one of the curious features between particles physics and cosmology. These features are the possibility of obtaining cosmological large numbers, as mass $M_{U}$ radius $R_{U}$ and age $T$ of the universe, scaling up the typical values of mass $m$, size $r$ and life time $t$ appearing in particle physics, by the scale factor $10^{38-40}$. The scale relations are $T / t \sim R_{U} / r \sim\left(M_{U} / m\right)^{1 / 2} \sim$ $\lambda=10^{38-40}$. From here we can scale $h$ in order to obtain the new constant $\mathcal{H}$ of the new scale invariance of quantum mechanics. From a simple dimensional analysis we have $\mathcal{H} \sim \lambda^{3} h$. The possible meaning of this new constant $\mathcal{H}$ is that $\mathcal{H} /(2 \pi)$ is the angular momentum of a rotating universe and this explanation is close to the Gödel's spin, with the Kerr limit for the spin, and with the Muradian's Regge-like relation for galaxies and clusters, see Carneiro (1998) and references therein. In fact this new constant is $\mathcal{H} \sim 10^{120} h$ and is what is call in Alfonso-Faus (2008) the cosmological Planck's constant. With this new Planck's constant no large numbers appear at the cosmological level. In Carneiro (1998) it is also described an intermediate scale invariance of quantization related to the angular momenta of stars and close to the Kerr limit for a rotating black hole with mass around $10^{30} \mathrm{~kg}$. All these ideas suggest treating the universe as a single particle, as we shall see later. In fact as a cosmological quantum black hole. In the following sections we will see that several scaling laws can explain some of the present cosmological problems. 


\section{The Large number coincidence problem}

Hermann Weyl (1917, 1919) speculated that the observed radius of the universe might also be the hypothetical radius of a particle whose energy $m_{h} c^{2}$ is equals to the gravitational self-energy of the electron $G m_{e}^{2} / r_{e}$, where $m_{h}$ is the mass of the hypothetical particle, $m_{e}$ and $r_{e}$ the mass and the radius of the electron. This was the beginning of the large number coincidence problem. Hence, we have

$\frac{R_{U}}{r_{e}} \sim \frac{r_{h}}{r_{e}}=\frac{m_{e}}{m_{h}}=\frac{m_{e}}{\frac{G m_{e}^{2}}{c^{2} r_{e}}}=\frac{c^{2} r_{e}}{G m_{e}}=\frac{e^{2}}{4 \pi \varepsilon_{0} G m_{e}^{2}} \approx 10^{42}$

where we have that $r_{e}=e^{2} /\left(4 \pi \varepsilon_{0} m_{e} c^{2}\right)$ and $r_{h}=$ $e^{2} /\left(4 \pi \varepsilon_{0} m_{h} c^{2}\right)$. This coincidence was further developed by Eddington (1931) who related the above ratios with $N$, the estimated number of charged particles in the universe.

$\frac{e^{2}}{4 \pi \varepsilon_{0} G m_{e}^{2}} \sim \sqrt{N} \approx 10^{42}$.

Eddington obtained the most intriguing relation between the present number of baryons in the universe, known as the Eddington number, and the squared ratio of the electric to the gravitational force between the proton and the electron.

$\frac{F_{e}}{F_{g}}=\frac{e^{2}}{4 \pi \varepsilon_{0} G m_{e} m_{p}} \sim \frac{c T}{r_{e}} \approx 10^{40}$

where $m_{p}$ is the proton mass and $T$ is the age of the universe. This coincidence between large numbers can also be expressed in the alternative form

$\hbar^{2} H_{0} \sim G m_{n}^{3} c$

where $m_{n}$ is the nucleon mass, $H \equiv \dot{a} / a$ is the Hubble parameter and $H_{0}$ is its present value and $a(t)$ is the scale factor, see Mena Marugan \& Carneiro (2002). This approximate identity is called the EddingtonWeinberg relation. The Hubble parameter is not a constant and varies as the inverse of the cosmological time $t$ in the standard Friedmann-Robertson-Walker (FRW) cosmology. This fact led Dirad 1937, 1978) to speculate the hypothesis that Newton's constant $G$ must depend on time as $H$, i.e. $G \sim 1 / t$, so that relation (1) remains always valid. This fact is incompatible with the experimental bounds that exist on time variation of $G$, see Damour et al. (1988); Mena Marugan \& Carneiro (2002); Williams et al. (1996). Hence, the coincidence (11) is only valid in this epoch. In Funkhouser (2006) it was resolved the large number coincidence problem using scaling laws from the standard cosmological model.
We reproduce here the arguments. From the scale relations (that do not constitute a coincidence problem)

$\left(\frac{M_{U}}{m_{n}}\right)^{1 / 2} \sim\left(\frac{m_{P}}{m_{n}}\right)^{2} \sim\left(\frac{M_{U}}{m_{P}}\right)^{2 / 3}$,

where $m_{P}$ is the Planck mass, we obtain $M_{U} \sim m_{P}^{4} / m_{n}^{3}$ where $M_{U}=\Omega_{m}(4 \pi / 3) R_{U}^{3} \rho_{c}=(4 \pi / 3) R_{U}^{3} \rho_{m}$ is the observable mass of the universe. The Hubble parameter in a universe with zero curvature is related with the average total energy density $\varepsilon$ by

$H^{2}=\frac{8 \pi G \varepsilon}{3 c^{2}}$,

and during the matter-dominance the total energy is $\varepsilon=c^{2} \rho_{m}$. Therefore the mass of the universe is equals

$M_{U}=\frac{4 \pi}{3} R_{U}^{3} \rho_{m}=\frac{R_{U}^{3} H^{2}}{2 G}$.

Taking into account that $H \sim c / R_{U} \sim 1 / T$ equation (3) gives the scaling law

$G M_{U} \sim c^{2} R_{U}$

Expression (4) was obtained by Whintrow (1946), Whintrow \& Randall (1951), Sciama (1953), Brans \& Dicke (1961); Dicke (1961) and also by Assis (1989, 1999) in different contexts. Another form to obtain equation (4) is applying the classical Mach's principle by requiring that the self-energy of a body is given by the gravitational energy of interaction of a body with the whole universe:

$m c^{2}=\frac{G m M_{U}}{R_{U}}$.

Substituting this scaling law (4) in the expression $M_{U}=m_{P}^{4} / m_{n}^{3}$ and remembering that the Planck mass is $m_{P}=\sqrt{\hbar c / G}$ we have

$\frac{c^{2} R_{U}}{G}=\frac{m_{P}^{4}}{m_{n}^{3}}=\frac{\hbar^{2} c^{2}}{G^{2} m_{n}^{3}}$,

and from here the Eddington-Weinberg relation (1).

\section{The Cosmic coincidence problem}

In an expanding universe with scale factor $a(t)$, where $t$ is the cosmological time, $\Lambda$ is a constant while the matter density $\rho_{m}$ decreases with $a^{3}$. However, the observed energy density of matter $c^{2} \Omega_{m} \rho_{c}$ is so close to the vacuum energy density attributed to the cosmological 
constant $\Lambda$, given by $\varepsilon_{v a c}=3 \Lambda c^{2} /(8 \pi G)$. This coincidence is known as the cosmic coincidence problem and may be expressed as

$\rho_{m}=\Omega_{m} \rho_{c} \sim \frac{3 \Lambda}{8 \pi G}$.

As in the case of the large number coincidence, this coincidence occur only in this epoch. We are going to see that the cosmic coincidence problem is a consequence of the large number coincidence and due to the fact that we are in the era of vacuum-dominance. If we assume that the present evolution of the universe is dominated by the cosmological constant $\Lambda$, as corroborated by observation Tegmark et al. (2001), we can set $H_{0} \sim \Lambda^{1 / 2}$. The continuous transition from the matter-dominance given by equation (2) to our era of vacuum-dominance gives the cosmic coincidence

$H_{0}^{2} \sim \frac{8 \pi G \rho_{m}}{3} \sim \Lambda$

\section{The Cosmological constant problem}

If $\Lambda$ originates from the vacuum quantum fluctuations, its theoretically expected value has order of $l_{p}^{-2}$ where $l_{p} \equiv \sqrt{\hbar G / c^{3}} \approx 10^{-35} \mathrm{~m}$ is the Planck length, see Weinberg (1989). That is, 122 orders of magnitude greater than the observed value $\Lambda \approx 10^{-52} \mathrm{~m}^{-2}$, see Tegmark et al. (2001). This huge discrepancy is known as the cosmological constant problem and it is an open problem nowadays, see for instance Weinbero (1989); 't Hooft \& Nobbenhuis (2006).

However we can get for the cosmological constant $\Lambda$ one scaling law that also explains the cosmic coincidence, see Funkhouser (2006, 2008). Putting the condition $H_{0} \sim \Lambda^{1 / 2}$ in the large number coincidence (1) we have

$\Lambda \sim \frac{G^{2} m_{n}^{6} c^{2}}{\hbar^{4}}$.

Equation (5) is essentially the same scaling law derived by Zel'dovich (1967), from considerations of field theory and empirical arguments. This form to derive equation (5) was first made by Matthews (1998), who takes relation (11), as well as the present dominance of the cosmological constant over the density of matter. Taking into account that the Compton wavelength of the nucleon is $\lambda_{n}=h /\left(m_{n} c\right)$ and the scale relation

$\left(\frac{M_{U}}{m_{n}}\right)^{1 / 2} \sim \frac{R_{U}}{\lambda_{n}}$ from (5) we obtain the scaling laws

$c^{2} \Lambda \sim \frac{G^{2} m_{n}^{2}}{\lambda_{n}^{4}} \sim \frac{G^{2} M_{U}^{2}}{R_{U}^{4}}$.

This scaling law says that the energy density associated to the cosmological constant may be scaled to the gravitational energy of the nucleon mass confined to a sphere whose radius is the Compton wavelength of the nucleon and to the gravitational energy of the universe of mass $M_{U}$ and whose radius is $R_{U}$. This is the generalization of the Zel'dovich (1967) equation (5) to the cosmological level

$\Lambda \sim \frac{G^{2} M_{U}^{6} c^{2}}{\mathcal{H}^{4}}$,

with the introduction of the cosmological Planck's constant $\mathcal{H}$ satisfying $R_{U}=\mathcal{H} /\left(M_{U} c\right)$ and the generalization of the Eddington-Weinberg relation (1)

$\mathcal{H} H_{0} \sim G M_{U}^{3} c$,

assuming that the present evolution of the universe is dominated by the cosmological constant $\Lambda$ and then we have $H_{0} \sim \Lambda^{1 / 2}$. These generalizations are also obtained in Alfonso-Faus (2008, 2011). Moreover the cosmological constant problem is solved with the introduction of the cosmological Planck's constant $\mathcal{H}$ because now $\Lambda_{c}$ originates from the cosmological vacuum quantum fluctuations, has the value of order $L_{p}^{-2}$ where $L_{p} \equiv \sqrt{\mathcal{H} G / c^{3}} \approx 10^{26} m$ is the cosmological Planck length, and we obtain $\Lambda_{c} \approx 10^{-52} \mathrm{~m}^{-2}$ which agrees with the observed value. In fact, this cosmological Planck length $L_{p}$ is of order of the radius of the universe $R_{U}$. Hence we have $R_{U}^{2} \sim \mathcal{H} G / c^{3}$, that taking into account $R_{U}=\mathcal{H} /\left(M_{U} c\right)$ we reobtain the equality (4) that relates $M_{U}$ with $R_{U}$. In resume we have the following identities that define the cosmological scale $R_{U}=G M_{U} / c^{2}$, the cosmological Compton wavelength $\bar{\lambda}_{c}=\mathcal{H} /\left(M_{U} c\right)$, the new cosmological constant $\Lambda_{c} \sim L_{p}^{-2} \sim R_{U}^{-2}$ and it is satisfied that $\Lambda_{c} \mathcal{H}=c^{3} / G$. Hence we have two important scales, the micro scale called Planck scale and the macro scale given by the cosmological scale that suggest the scale relativity invariance introduced by Nottale (1992).

\section{The critical acceleration coincidence}

The observed motions of clusters of galaxies and material within galaxies may be interpreted to indicate that the laws of dynamics deviate from Newtonian models at accelerations smaller than some critical acceleration 
$a_{0} \approx 10^{-10} \mathrm{~ms}^{-2}$, see Milgrom (1983). The Hubble acceleration $\mathrm{cH}_{0}$ is of the same order only in this epoch. This coincidence $a_{0} \sim c H_{0}$ is well known from the first works of Milgrom (1983) see also Funkhouser (2006). This coincidence is justified in Giné (2009) and Giné (2011) by different arguments. Substituting $H_{0} \sim \Lambda^{1 / 2}$ the coincidence takes the form $a_{0} \sim c \Lambda^{1 / 2}$ and taking into account the scaling law (7) we obtain

$a_{0} \sim \frac{G m_{n}}{\lambda_{n}^{2}}$.

Hence, the critical acceleration is scaled to the characteristic gravitational acceleration of the nucleon mass at its Compton length. Moreover, taking into account the scale relation (6) we have that

$a_{0} \sim \frac{G M_{U}}{R_{U}^{2}}$.

Hence, the critical acceleration is scaled to the characteristic gravitational acceleration of any body in our universe due to the all the rest of the mass of the universe. This interpretation of the critical acceleration appears in Gine (2009) in the context of a implementation of the inertia Mach's theory. In Tank (2010, 2011) it is found that identity (10) is invariant at any scale because is satisfied by the hadrons, the electrons, the nucleus, the globular clusters, the galaxies, the clusters of galaxies, the universe as a whole and others physical situations.

\section{The cosmic acceleration problem}

The standard candle observations of type Ia supernovae give a cosmic acceleration with a positive rate, which implies the introduction of the cosmological constant in the cosmological models. Hence, the expansion of the universe is accelerating, see Riess et al. (1998). This acceleration states the cosmic acceleration problem. The question is what causes this acceleration?

It is clear that the introduction of the cosmological constant give as a consequence that the universe is accelerating. However, what is the nature of this cosmological constant introduced?

There are essentially two ways of introducing the cosmological constant or the dark energy. The first one is changing gravitation with $f(R)$ gravity models, Scalartensor models, braneworld models, etc. The second one is changing matter with the quintessence, K-essence, tachyons, Chaplygin gas, phantom field, etc.

We have seen that the Eddington-Weinberg relation (11) is only valid in this epoch. However, the strong version of the cosmological holography principle also implies equation (1), see Carneiro (2002), without any additional assumption as the dominance of the cosmological constant $\Lambda$. Hence, in this case the relation (11) is valid for any cosmological time. The derivative respect to the time of the relation (11) gives $\dot{H}=0$, because the variation of $G$ is incompatible with the observations. Now, we recall the definition of the deceleration parameter $q=-a \ddot{a} / \dot{a}^{2}$ and its relation with the Hubble parameter

$\dot{H}=-(1+q) H^{2}$.

Therefore, the strong version of the cosmological holography principle implies that $q \approx-1$ in order to obtain $\dot{H}=0$. This value of the deceleration parameter is also found in the context of the modified Newtonian theory (MOND) in Gine (2010), when we evaluate the recessional acceleration $a_{r}(t)=-q H v_{r}$ for the objects receding from us at a rate faster than the speed of light and compare with the value of the constant acceleration $a_{0}=H_{0} c$. In this case the Hubble law is applied for close distances assuming the same behavior at first order for largest observable distance.

\section{The quantum universe}

We have seen the existence of several scaling laws that explain some of the present cosmological problems. However, the origin of the dark energy and dark matter are still open problems.

In Alfonso-Faus (2011) it is given a necessary and sufficient condition for an object of any mass $m$ to be a quantum black hole generalizing the results obtained for the cosmological scale. This generalization is established by the following identities that define a quantum black hole for each $m$ and a new scale. The first is $r_{m}=G m / c^{2}$, where $r_{m}$ is the gravitational radius, the generalized Compton wavelength $\bar{\lambda}_{m}=h_{m} /(m c) \sim r_{m}$, where $h_{m}$ is the generalized Planck's constant, the $\Lambda_{m} \sim r_{m}^{-2}$ and it is satisfied that $\Lambda_{m} h_{m}=c^{3} / G$. This generalization is also justified by the described intermediate scale invariance of quantization for a rotating black hole with certain mass, see Carneiro (1998). Hence in Alfonso-Faus (2011); Fullana \& Alfonso-Faus (2011) is adopted the idea that the universe is a quantum black hole and therefore it is possible to define, following the Hawking (1975) formulation, the entropy of the universe as a quantum black hole

$S=\frac{4 \pi k_{B}}{\hbar c} G M^{2}=\pi k_{B}\left(\frac{R_{U}}{l_{p}}\right)^{2} \approx 10^{122} k_{B}$,

which is in accordance with the current value found by Egan \& Lineweaver (2010). In Fullana \& Alfonso-Faus 
(2011) it is computed the conjugate black hole of the universe that is identified with the quantum of the gravitational potential field and the bit. Besides, the information-entropy relation, based on the bit, the Padmanabhan (2010a, b) proposal that gravity has an entropic or thermodynamic origin, and the Verlinde (2011) interpretation of gravity as an emerging entropic force, gives a hope to unify gravity with quantum theory.

The idea of Alfonso-Faus \& Fullana reinforces the relationships between the constants of atomic physics and the constants of the Universe, as we have described in the text, see also Haidukovic (2010a); Dinculescu (2009). In Hajdukovid (2010a) three interesting relations are presented. The first one connects the Compton wavelength of a pion and the dark energy density of the universe; the second one connects the Compton wavelength of a pion and the mass distribution of nonbaryonic dark matter in a galaxy; the third one relates the mass of a pion to fundamental physical constants and cosmological parameters which has as particular case the Eddington-Weinberg relation (10) but for the pion mass. The importance of the pions (instead of the nucleon mass) is due to "virtual" pions, which are, accoding to quantum field theory, an inherent part of vacuum fluctuations and as a simple particles (quark pairs) dominate the quantum vacuum. We recall that pions are the subatomic particles that describe the interaction between nucleons. Under this scenario, each nucleon is continuously emitting and reabsorbing virtual pions, which surround it like a swarm. Moreover correct value of mass to put in the identity (5) according to the observed value of $\Lambda$ is about 1/20 times the proton mass or about 80 times the electron mass and is about one third the pion mass, see Santos (2010). Therefore the pions must dominate the quantum vacuum fluctuations that contribute to the value of the cosmological constant. In Dinculescu (2009) it is derived the values of the baryon density parameter, the Hubble constant, the cosmic microwave background temperature and the helium mass fraction in excellent agreement with the the most recent observational data.

Following the idea of a quantum vacuum fluctuations, with virtual particles flashing in and out of existence, in Santos (2010, 2011) it is showed that the vacuum fluctuations effectively supplies a vacuum energy pressure which is of the right order of magnitude to explain dark energy. The key idea of the Santos works is the two-point correlation function of vacuum fluctuations gives the correct contribution of Dark energy, and this relies upon the disappearance of the correlation within the Planck length which solves the cosmological constant problem.
The following exciting papers papers can shed light on the nature of the dark matter and the solution of the dark matter problem. In Villata (2011) showed that, from $\mathrm{CPT}$ invariance of the general relativity, the sign of the gravitational force between matter and antimatter is reversed (anti-gravity). This is a controversial result which is being analyzed and discussed, see for instance Cabbolet (2011); Cross (2011) and Villata (2011b).

Based in the anti-gravity (that a particle and its antiparticle have the gravitational charge of the opposite sign) Hajdukovic (2011a,b) consider that the quantum vacuum may be considered as a fluid of virtual gravitational dipoles. In such a way that when we place a gravitational mass in a quantum vacuum will induce a polarization of the quantum vacuum, in the same way that a charge induces polarization in a surrounding dielectric medium. In the case of gravitation, we would expect to find more virtual particles close to a gravitating object, and more anti-particles at much greater distance. This would mean that, in a galaxy for example, the apparent gravitational attraction of the body is an increasing function of distance out to some critical value. Following this hypothesis, Hajdukovic present the first indications that dark matter may not exist and that the phenomena for which it was invoked might be explained by the gravitational polarization of the quantum vacuum by the known baryonic matter. The best developed alternative to particle dark matter is the Modified Newtonian Dynamics (MOND) Milgrom (1983), but we witness a violation of the fundamental law of gravity and has still fundamental problems with the observational data, see for instance Giné (2010, 2011b) and references therein. However in the Hajdukovic model the distribution of vacuum polarization will depend on the distribution of matter, so the apparent extra acceleration towards the center of mass will vary from one object to another, and as a function of position within the object, see Hajdukovic (2010b). Moreover the consequences of the model can be tested, see Hajdukovid (2011b), where some phenomena partially explained by dark matter and theories of modified gravity are understood in the framework of the gravitational polarization. Moreover the theory presented in Hajdukovic (2011a,b) is not a support to MOND although there is a critical gravitational filed which corresponds to the maximal gravitational polarization density.

The final conclusion is that is needed a quantum gravitational theory with a quantum granulation of space-time and in this new framework the presented papers have given us grounds to hope that both dark 
energy and dark matter will find their natural explanation as simply naturally-arising quantum vacuum phenomena.

Acknowledgements The author is partially supported by a MICINN/FEDER grant number MTM2011 -22877 and by a Generalitat de Catalunya grant number 2009SGR 381 


\section{References}

Alfonso-Faus, A. 2008, Astrophys. Space Sci., 318, 117

Alfonso-Faus, A. 2011, Astrophys. Space Sci., 325, 113

Assis, A.K.T. 1989, Found. Phys. Lett., 2, 301

Assis, A.K.T. 1999, Relational Mechanics, Apeiron, Montreal

Brans, C. \& Dicke, R.H. 1961 Phys. Rev. 124, 925

Cabbolet, M.J.T.F. 2011, Astrophys. Space Sci., in press, arXiv: 1108.4543

Caldirola, P., Pavsic, M. \& Recami, E. 1978, Nuovo Cimento, 48 B, 205

Carneiro, S. 1998, Found. Phys. Lett., 11, 95

Carneiro, S. 2002, preprint, arXiv:gr-qc/0206064v1

Cross, D.J. 2011, arXiv:1108.5117

Damour, T., Gibbons, G.W. \& Taylor, J.H. 1988, Phys. Rev. Lett., 61, 1151

Dicke, R.H. 1961, Phys. Rev. Lett., 7, 359

Dinculescu, A., 2009 Astrophys. Space Sci., 323, 289

Dirac, P.A.M. 1937, Nature 139, 323

Dirac, P.A.M. 1978, Directions in physics. In WileyInterscience, New York, 7286

Eddington, A.S. 1931, Proc. Camb. Phil. Soc. 27, 15

Egan, C.A. \& Lineweaver, C.H. 2010, Astrophys. J., 710, 1825

Fullana, M.J. \& Alfonso-Faus, A. 2011, Astrophys. Space Sci., in press, DOI 10.1007/s10509-011-0909-1

Funkhouser, S. 2006, Proc. R. Soc. A 462, 3657

Funkhouser, S. 2008, Proc. R. Soc. A 464, 1345

Giné, J. 2009, Chaos Solitons Fractals, 41, 1651

Giné, J. 2010, preprint, UdL, http://web.udl.es/usuaris /t4088454/ssd/Prepublicaciones/PS/mond.pdf

Giné, J. 2011, Internat. J. Theoret. Phys, 50, 607

Giné, J. 2011b, Astrophys. Space Sci., in press, arXiv:1108. 3565

Hajdukovic, D.S. 2010a, Astrophys. Space Sci., 326, 3

Hajdukovic, D.S. 2010b, Astrophys. Space Sci., 330, 207

Hajdukovic, D.S. 2011a, Astrophys. Space Sci., 334, 215

Hajdukovic, D.S. 2011b, Astrophys. Space Sci., in press, arXiv:1111.4884

Hawking, S.W. 1975, Commum. Math: Phys., 43, 199

't Hooft, G. \& Nobbenhuis S. 2006, Class. Quantum Gravity, 23,3819

Matthews, R.A.J. 1998, Astronomy \& Geophysics 39, 19

Mena Marugan, G.A. \& Carneiro, S. 2002, Phys. Rev. D, 65, 087303

Milgrom, M. Astrophys. J., 270, 365

Nottale, L. 1992, Internat. J. Modern Phys. A, 7, 4899

Padmanabhan, T. 2010a, Rep. Prog. Phys., 73, 046901

Padmanabhan, T. 2010b, Mod. Phys. Lett. A, 25, 1129

Riess, A. et al. 1998, Astronomical J. 116, 1009

Salam. A. \& Strathdee, J. 1977, Phys. Rev. D, 16, 2668

Salam. A. \& Strathdee, J. 1978, Phys. Rev. D, 18, 4480

Santos, E. 2010, Astrophys. Space Sci., 326, 7

Santos, E. 2011, Astrophys. Space Sci., 332, 423

Sciama, D.W. 1953, Mon. Not. R. Astron. Soc., 113, 34

Sivaram, C. \& Sinha, K.P. 1979 Phys. Rep., 51. 111

Tank, H.K. 2010 Astrophys. Space Sci., 330, 203

Tank, H.K. 2011 Astrophys. Space Sci., 336, 341

Tegmark, M., Zaldarriaga, M., \& Hamilton, A.J.S. 2001, Phys. Rev. D, 63, 043007

Verlinde, E. 2011, J. High Energy Phys., 4, 29
Villata, M. 2011, EPL (Europhysics Letters), 94, 2011

Villata, M 2011b, Astrophys. Space Sci., in press, arXiv: 1109.1201

Weinberg, S. 1989, Rev. Mod. Phys. 61, 1

Weyl, H. 1917, Ann. Phys., 54, 117

Weyl, H. 1919, Ann. Phys., 59, 101

Whitrow, G.J. 1946, Nature, 158, 165

Whitrow, G.J. \& Randall, D.G. 1951, Mon. Not. R. Astron. Soc., 111, 455

Williams, J.G., Newhall, X.X. \& Dickey, J.O. 1996, Phys. Rev. D, 53, 6730

Zeldovich, Ya.B. 1967, Sov. Phys. JETP Lett., 6, 316 